\title{
Pursuit of Oxidation Behavior for Conjugated Polyenoyl Glycerols and Establishment of Their Novel Oxidation Prevention Method
}

\author{
Yohko Kuge, Ayato Kanda and Setsuko Hara* \\ Department of Materials and Life Science, Faculty of Science and Technology, Seikei University (3-1 Kichijoji-kitamachi 3, Musashino-shi, \\ Tokyo 180-8633, JAPAN)
}

\begin{abstract}
Although conjugated oils are paid much attentions to their interesting physiological properties such as anticancer, anti-arteriosclerosis, anti-hypertension activities, loss in body fat etc, there is few information on their oxidation behavior. In the present work, their oxidation behavior and oxidation prevention method were evaluated to utilize as functional foods or drugs.

As results, an oxidation behavior of conjugated oils was different from that of corresponding nonconjugated oils, and conjugated oils were supposed to form not only hydroperoxides but also kinds of cyclic peroxides as primary oxidation products in the autoxidation.

In a thermal oxidation, polymerization reaction might be prior to decomposition reaction owing to form a large quantity of more polymerized products in conjugated oils. Solidification of conjugated oils by thermal oxidation was prevented for long time by addition of tocopherol, and optimal addition amounts of tocopherol into conjugated oils were $1,000 \mathrm{ppm}$ either in autoxidation or thermal oxidation. Equi-molar of phosphatidyl ethanolamine showed synergistic effect slightly on $1,000 \mathrm{ppm}$ tocopherol for preventing thermal oxidation of conjugated oil.
\end{abstract}

Key words: conjugated linoleic acid, conjugated linolenic acid, autoxidation, thermal oxidation, oxidation prevention, antioxidant, synergist

\section{INTRODUCTION}

Conjugated linoleic acids (CLA) have various physiologic activities, including antiobesity, anticancer, antiatherogenic, immunostimulatory and antihyperlipidemic effects ${ }^{1-7)}$. The putative mechanism underlying the decrease in body fat induced by CLA involves various factors, such as the acceleration of lipid degradation in fat cells, increased fat metabolism/energy consumption, inhibition of fat cell multiplication, reduction in fat cell size, and apoptosis induction. Regarding the anticancer effect of CLA, it has been reported that the diet supplementation with $0.1-1 \%$ CLA inhibits the induction of chemical carcinogenesis in rats and mice ${ }^{8}$. Furthermore, in experiments using cultured cells, it has been shown that CLA inhibits the growth of cells derived from human breast cancer, cancer of the large intestine, and malignant melanoma ${ }^{9}$. The feeding of a diet that contained $0.5 \%$ CLA to mice for 3 weeks caused their immunoglobulin levels to increase and their cytokine pro- duction levels to decrease, which confirmed the immunomodulatory effect of CLA ${ }^{10}$.

Conjugated fatty acids are mainly present in vegetable seed oils, and a conjugated trienoic fatty acid, $\alpha$-eleostearic acid, accounts for about $80 \%$ of all the fatty acids in tung oil and about $60 \%$ of all the fatty acids present in bitter gourd (Momordica charantia) seed oil. Garden balsam seed oil contains parinaric acid, which is a conjugated tetraenoic fatty acid. Marine algae contain very low levels of fatty acids with $\mathrm{C} 20$ - and C22-conjugated trienoic and tetraenoic structures ${ }^{11)}$. Most importantly, conjugated linolenic acids (CLN) are reported to exert more potent cytotoxic effects than CLA on cancers of the large intestine, liver, breast, stomach, and lung ${ }^{12)}$.

As described above, conjugated fatty acids with various physiologic activities are expected to have applications in foodstuffs and medicines. Therefore, to utilize conjugated fatty acids in safe and effective manners in foodstuffs, it is

*Correspondence to: Setsuko Hara, Department of Materials and Life Science, Faculty of Science and Technology, Seikei University, 3-1 Kichijoji-kitamachi 3, Musashino-shi, Tokyo 180-8633, JAPAN

E-mail: shara@st.seikei.ac.jp

Accepted February 22, 2009 (recieved for review December 16, 2008)

Journal of Oleo Science ISSN 1345-8957 print / ISSN 1347-3352 online

http://www.jstage.jst.go.jp/browse/jos/ 


\section{Y. Kuge, A. Kanda and S. Hara}

necessary to elucidate the oxidative stabilities of conjugated fatty acids and to establish an effective antioxidant method for employing CLA and CLN. To date, there have been several reports on the oxidative stabilities of conjugated fatty acids. Suzuki et al..$^{13)}$ reported that the autoxidative stabilities of CLA-TAG (containing 34.30\% 9c,11tCLA and 35.2\% 10t,12c-CLA) and bitter gourd (Momordica charantia) seed oil (containing about $61.6 \% 9 c, 11 t, 13 t$-CLN) were lower than those of soybean oil and perilla oil, which are nonconjugated oils. Moreover, Lee et $a l .^{14)}$ reported that $t$-butylhydroquinone conferred the most potent antioxidant activity on CLA of the seven synthetic antioxidants.

In the present study, we examined the oxidation behaviors of conjugated polyenoyl glycerols (conjugated oils) in non-aqueous and emulsifying autoxidation tests and a thermal oxidation test, using a non-conjugated fat as the control. In addition, we examined the antioxidant effects of tocopherol (Toc), as well as those of phosphatidylethanolamine (PE), ascorbyl palmitate (AP) and citric acid (CA), which are synergists for Toc.

\section{EXPERIMENTAL}

\subsection{Samples and reagents}

The following oils were used: conjugated oils, CLA-TAG (The Nisshin OilliO Group, Tokyo, Japan) and CLN-TAG (tung oil; Hayashi Chemical Industry Co., Kyoto, Japan); and non-conjugated oils, high-linoleic safflower oil (LATAG; The Nisshin OilliO Group) and linseed oil (LN-TAG; Hayashi Chemical Industry). As an antioxidant, mix Toc (The Nisshin OilliO Group) was used, and as synergists, L(+)AP, (Wako Pure Chemical Industries, Ltd., Osaka, Japan) and CA (a special-grade reagent; Kokusan Chemical Co., Tokyo, Japan) were used. High-purity PE was purified by silica-gel column fractionation from LS-60 (PC-70), which is a purified soybean phospholipid produced by The Nisshin OilliO Group. In the emulsifying autoxidation test, Tween ${ }^{*} 20$ (Koso Chemical Co., Tokyo, Japan) was used. Regarding the remaining reagents, special-grade, commercially available reagents were used without modification.

\subsection{Evaluation of oxidative stability}

2.2.1 Purification of sample oils

Sample oils were used following purification for the removal of Toc and peroxides through silica-gel column fractionation using a hexane:diethyl ether mixture at 85:15 $(\mathrm{v} / \mathrm{v})$ as a elution solvent.

2.2.2 Non-aqueous autoxidation test

To reconfirm the report by Suzuki et al. ${ }^{13)}$ in non-aqueous autoxidation test, CLA-TAG, LA-TAG, CLN-TAG, and LN-TAG (Table 1) were incubated in a $30^{\circ} \mathrm{C}$ thermostatic chamber for autoxidation in the dark and under fluorescent light irradiation at 960 lux. The hydroperoxide, carbonyl
Table1 Characterization of Sample Oils Used in the Present Study.

\begin{tabular}{|c|c|c|c|c|c|}
\hline & $\begin{array}{l}\text { CLA- } \\
\text { TAG }\end{array}$ & $\begin{array}{l}\text { LA- } \\
\text { TAG }\end{array}$ & $\begin{array}{c}\text { CLN- } \\
\text { TAG }\end{array}$ & $\begin{array}{l}\text { LN- } \\
\text { TAG }\end{array}$ \\
\hline \multirow{7}{*}{ 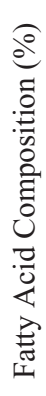 } & Palmitic & 6.8 & 9.1 & 4.6 & 8.9 \\
\hline & Stearic & 2.2 & 2.1 & 4.2 & 3.5 \\
\hline & Oleic & 10.2 & 14.4 & 11.2 & 21.0 \\
\hline & Linoleic & 0.9 & 74.4 & 11.4 & 17.0 \\
\hline & $\alpha$-Linolenic & - & - & - & 49.6 \\
\hline & CLA & 79.9 & - & - & - \\
\hline & CLN & - & - & 68.6 & - \\
\hline \multicolumn{2}{|c|}{ TAG Purity (\%) } & 100.0 & 100.0 & 100.0 & 100.0 \\
\hline \multicolumn{2}{|c|}{ PV (meq/kg) } & 1.0 & 1.1 & 0.8 & 10.1 \\
\hline \multicolumn{2}{|c|}{$\mathrm{AnV}$} & 1.3 & 0.1 & 7.3 & 0.5 \\
\hline \multicolumn{2}{|c|}{ Toc Content (ppm) } & 0 & 0 & 0 & 0 \\
\hline
\end{tabular}

compound, and polar compound (PC), polymer contents were measured over time using potentiometric titration of the peroxide value (PV), colorimetric determination of anisidine value (AnV), TLC-FID and GPC analyses, respectively.

\subsubsection{Emulsifying autoxidation test}

Tween ${ }^{\#} 20$ (5 wt\%) was added to CLN-TAG and LN-TAG, and an autoxidation test was conducted in a $30^{\circ} \mathrm{C}$ thermostatic chamber as follows: type $\mathrm{O} / \mathrm{W}(3 / 7)$ and type W/O $(3 / 7)$ emulsified samples, which were prepared by homogenization at 8,000 rpm for $20 \mathrm{~min}$, shaking and stirring at 1,000 rpm. The emulsified samples were sampled over time, and the lipids were recovered. The PV, AnV, and PC content were measured.

\subsubsection{Thermal oxidation tests}

In thermal oxidation test I, CLA-TAG and LA-TAG were heated continuously for $6 \mathrm{~h}$ to $60,90,120,150$ and $180^{\circ} \mathrm{C}$ using the Rancimat apparatus (Metrohm AG, Herisau, Switzerland), and samples were taken every hour. In thermal oxidation test II, CLN-TAG and LN-TAG were heated continuously for $3 \mathrm{~h}$ to $60,90,120,150$ and $180^{\circ} \mathrm{C}$, and samples were taken every $30 \mathrm{~min}$. To determine the changes over time in the properties related to the thermal oxidation tests, the AnV, Toc residual ratio, residual ratios of major fatty acids were measured, as well as the amounts of polymeric substances and PC content, as appropriate. 2.2.5 Antioxidant techniques for conjugated oils

In antioxidant technique test I, with the aim of examining the antioxidant effect of Toc, 0-2,000 ppm Toc were added to CLA-TAG and CLN-TAG. In antioxidant technique test II, synergists (PE, AP, CA) were mixed with 
1,000 ppm Toc, which was determined using antioxidant technique I as the optimal level, at a Toc-to-synergist molar ratio of 1:1, to examine the synergistic effects. In antioxidant techniques I and II, the samples were heated at $100^{\circ} \mathrm{C}$ continuously for $8 \mathrm{~h}$ using the Rancimat apparatus. At $2 \mathrm{~h}$ intervals, polymers and PC contents were measured by GLC and TLC-FID analyses, respectively, and oxidative stability was evaluated according to the induction period in the Rancimat test (CDM test) at $100^{\circ} \mathrm{C}$.

\subsection{Analyses of sample composition}

\subsubsection{Lipid compositions (TLC-FID)}

The lipid compositions of CLA- and CLN-TAGs were determined by TLC-FID analysis using the IATROSCAN MK-6 (Mitsubishi Kagaku Iatron Inc., Tokyo, Japan). A developing solvent that consisted of benzene, chloroform, and acetic acid at a ratio of $35: 15: 1(\mathrm{v} / \mathrm{v} / \mathrm{v})$ was used.

2.3.2 Fatty acid compositions (GLC)

For the analysis of the fatty acid compositions of the oils, the constituent fatty acids were methyl-esterified ${ }^{15)}$, and a fused silica capillary column CBP1-S25-050 (0.32 mmI.D. $\times$ 25 m; Sinwa-kagaku Co., Ltd.) was connected to a GLC (model GC-18A; Shimadzu Corp., Kyoto, Japan) for the temperature programming GLC analysis. Column temperature was increased to $170-186^{\circ} \mathrm{C}\left(2^{\circ} \mathrm{C} / \mathrm{min}\right)$ and $186-200^{\circ} \mathrm{C}\left(7^{\circ} \mathrm{C}\right.$ $/ \mathrm{min}$ ), helium was used as the carrier gas, and FID was used as the detector.

2.3.3 Quantitative analysis of Toc (HPLC)

A normal-phase column Finepak SIL-5 (4.6 mmI.D. $\times 250$ mm; JASCO Corp.) was connected to an HPLC (model BIP1 ; JASCO), and a mobile-phase solvent that consisted of hexane and 2-propanol at a ratio of 124:1 was allowed to flow through the column at a rate of $0.7 \mathrm{~mL} / \mathrm{min}$. A fluorescence detector (model FP-2020 Plus, JASCO) with excitation at $295 \mathrm{~nm}$ and emission measured at $325 \mathrm{~nm}$ was used. For quantitative analysis, 2,2,5,7,8-pentamethyl-6-chromanol (PMC) was used as the internal standard, and the calibration curves prepared beforehand for Toc isomers (for $\alpha$-Toc, $\mathrm{y}=0.4508 \mathrm{x}-0.0927$; for $\beta$-Toc, $\mathrm{y}=0.7795 \mathrm{x}-0.3017$; for $\gamma$-Toc, $\mathrm{y}=1.2532 \mathrm{x}-0.7692$; and for $\delta$-Toc, $\mathrm{y}=1.2454 \mathrm{x}^{-}$ 0.9934 ) were used.

2.3.4 Quantitative analysis of polymeric substances (GPC)

Polymers that were produced as results of the autoxidation and thermal oxidation of oils were measured by GPC. GPC analysis was conducted by connecting a GPC KF802.5 column $(8.0 \mathrm{~mm}$ ID $\times 300 \mathrm{~mm}$; Showa Denko K.K.) and a differential refractometer (model RI-2031 Plus; JASCO) to a HPLC pump (model PU-2080 Plus; JASCO). As the mobile phase, tetrahydrofuran (THF) was allowed to flow through the column at a rate of $0.7 \mathrm{~mL} / \mathrm{min}$. In addition, calibration curves for the determination of molecular weights were prepared using high-linoleic safflower oil (LA-TAG) and tung oil as TAGs, Emulsy OL (C18:1-MAG; Riken Vitamin Co., Ltd.) as monoacylglycerol (MAG), oleic acid (Wako) as FA, and CLN-Me and LN-Me as fatty-acid methyl esters.

2.3.5 Measurements of oxidative degradation

PV-potentiometric titration, $\mathrm{AV}, \mathrm{AnV}$, and oxidizing acid measurements, polymeric substance measurements by GPC, kinetic viscosity measurements, and degree of staining measurements were performed in accordance with the standard method for the analysis of fats, oils and related materials prescribed by the Japan Oil Chemists' Society ${ }^{16)}$. Furthermore, PC content was determined by TLC-FID analysis using the method formulated previously ${ }^{17)}$.

\section{RESULTS AND DISCUSSION}

\subsection{Properties of samples}

Table 1 shows the properties of CLA-TAG, LA-TAG, CLN-TAG, and LN-TAG used in the present study, all of which except for the mixed TAG are available commercially. The CLA content of CLA-TAG was 79.9\%, the LA content of LA-TAG was $74.4 \%$, the CLN content of CLN-TAG was $68.6 \%$, and the LN content of LN-TAG was $49.6 \%$. Overall, the levels of TAG purity for all the samples were $100 \%$.

\subsection{Evaluation of autoxidation stability}

\subsubsection{Non-aqueous autoxidation test}

Measurements of the changes with time in PV, AnV, PC content, Toc residual ratio, and residual ratios of major fatty acids in the presence of CLA-TAG, LC-TAG, CLNTAG and LN-TAG revealed that PV increased to a greater extent in non-conjugated oil than in conjugated oil, although PC content was increased to a greater extent in conjugated oil than in non-conjugated oil.

Table 2 shows the AnV and PC content when the PV of each sample without Toc reached the value of $50 \mathrm{meq} / \mathrm{kg}$.

For the diene-type oils, the PV rose more rapidly in LATAG than in CLA-TAG, but concomitant with the rise in $\mathrm{PV}$, the AnV and PC content increased higher in CLA-TAG

Table 2 Comparison of $\mathrm{AnV}$ and $\mathrm{PC}$ Content at $\mathrm{PV}=50$ $\mathrm{meq} / \mathrm{kg}$ in Non-aqueous Autoxidation Tests at $30^{\circ} \mathrm{C}$ in the Dark and under Irradiation at 960 lux.

\begin{tabular}{|c|c|c|c|}
\hline & & $\mathrm{AnV}$ & PC content $(\%)$ \\
\hline \multirow{4}{*}{ 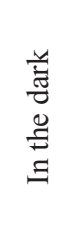 } & CLA-TAG & $15.2 \pm 1.1$ & $26.2 \pm 1.5$ \\
\hline & LA-TAG & $1.3 \pm 0.1$ & $3.1 \pm 0.2$ \\
\hline & CLN-TAG & $11.2 \pm 0.1$ & $26.0 \pm 1.4$ \\
\hline & LN-TAG & $0.8 \pm 0.1$ & $1.5 \pm 0.1$ \\
\hline \multirow{2}{*}{ 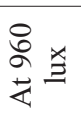 } & CLN-TAG & $24.0 \pm 1.3$ & $26.0 \pm 1.4$ \\
\hline & LN-TAG & $0.8 \pm 0.1$ & $1.5 \pm 0.1$ \\
\hline
\end{tabular}




\section{Y. Kuge, A. Kanda and S. Hara}

than in LA-TAG as shown in Table 2. In CLA-TAG, at 910 $\mathrm{h}$, at which point the PV was $133 \mathrm{meq} / \mathrm{kg}$, the surface became jelly-like, and the entire sample solidified with time, which meant that the oxidation state thereafter could not be evaluated accurately. Regarding the triene-type oils, at the start of autoxidation, the time course of PVs for CLN-TAG and LN-TAG were similar in CLA-TAG and LATAG. In CLN-TAG, solidification began at when the PV reached about $75 \mathrm{meq} / \mathrm{kg}$. Furthermore, comparison of the increments of $\mathrm{AnV}$ and $\mathrm{PC}$ content which contains polymerized compounds revealed that the formation of $\mathrm{PC}$ content in secondary oxidation products occurred more rapidly in conjugated oils than in non-conjugated oils. The information described above corresponds quite well with the results of Suzuki et al. ${ }^{13)}$, which means that dimmer and polymer contents increased more rapidly with time in conjugated oil than in non-conjugated oil.

Measurements of the changes over time in PV, AnV, and PC content following autoxidation of CLN-TAG and LNTAG under light irradiation at 960 lux and in the dark showed that PV increased to a greater extent in LN-TAG than in CLN-TAG. These results were different in AnV from those obtained in the dark. As shown in Table 2, when the PV was $50 \mathrm{meq} / \mathrm{kg}$, CLN-TAG produced more than twice the amount of carbonyl compounds under light irradiation as in the dark, whereas for LN-TAG, no increase in $\mathrm{AnV}$ was observed and the effect of illumination on PC content was negligible. These results were considered that the decomposition of CLN-TAG was accelerated under light irradiation to form carbonyl compounds.

Thus, it seems that the autoxidation of conjugated oils results in the production of carbonyl compounds and PC from both hydroperoxides and another type of peroxide such as a cyclic peroxide, while the autoxidation of nonconjugated oils results in the production of hydroperoxides as the primary oxidation products. Previously, it was presumed that PV did not increase significantly, since peroxides except hydroperoxides produced in conjugated oils were not measured. An another type of peroxide might be produced by 1,4-adition of oxygen molecule to conjugated diene moiety in conjugated oils to form a cyclic peroxide, and a cyclic peroxide thus produced might be decomposed to form secondary oxidation products such as carbonyl or polymeric compounds.

\subsection{Evaluation of the emulsifying autoxidation test}

Examination of the changes with time of PV, AnV and $\mathrm{PC}$ content in the $\mathrm{O} / \mathrm{W}$-type emulsifying autoxidation test revealed that $\mathrm{PV}$ increased to a greater extent in LN-TAG than in CLN-TAG, whereas the AnV and PC content increased to greater extents in CLN-TAG (Table 3).

Another examination of the oxidative behavior in the W/O-type emulsifying autoxidation test revealed that PV increased to a greater extent in LN-TAG than in CLN-
Table 3 Comparison of $\mathrm{AnV}$ and $\mathrm{PC}$ Content at $\mathrm{PV}=50$ $\mathrm{meq} / \mathrm{kg}$ between the $\mathrm{O} / \mathrm{W}$-type and W/O-type Emulsifying Autoxidation Tests at $30^{\circ} \mathrm{C}$ in the Dark.

\begin{tabular}{|c|c|c|}
\hline & AnV & PC content (\%) \\
\hline CLN-TAG (O/W) & $26.7 \pm 2.0$ & $20.0 \pm 1.4$ \\
\hline LN-TAG (O/W) & $2.1 \pm 0.1$ & $3.0 \pm 0.1$ \\
\hline CLN-TAG (W/O) & $33.3 \pm 2.3$ & $21.5 \pm 1.5$ \\
\hline LN-TAG (W/O) & $2.1 \pm 0.1$ & $3.0 \pm 0.1$ \\
\hline
\end{tabular}

TAG, whereas the increases in AnV and PC content when $\mathrm{PV}$ reached $50 \mathrm{meq} / \mathrm{kg}$ were greater in CLN-TAG (Table 3). Thus, this oxidative behavior is quite similar to that seen in the $\mathrm{O} / \mathrm{W}$-type emulsifying autoxidation test.

However, there were some differences in $\mathrm{AnV}$ and PC content between non-aqueous and emulsifying systems in autoxidation of CLN-TAG in the dark. It was presumed that carbonyl compound is formed more likely in emulsified oil than in non-aqueous oil because the minute oil particles in $\mathrm{O} / \mathrm{W}$ or $\mathrm{W} / \mathrm{O}$ emulsifying systems could contact with more oxygen molecules than neat oil, and the resulting peroxides like cyclic peroxides in emulsifying systems were decomposed to carbonyl compounds which were accumulated into PC.

\subsection{Evaluation of the thermal oxidation tests}

\subsubsection{Thermal oxidation test I}

CLA-TAG and LA-TAG were heated to $60-180^{\circ} \mathrm{C}$, and the polymer and $\mathrm{PC}$ contents increased in both samples as the temperature was increased. When both samples were heated to 120,150 and $180^{\circ} \mathrm{C}$, examination of the correlation (y $=\mathrm{ax}+\mathrm{b}$ ) between polymer content ( $\mathrm{x}$ axis) and $\mathrm{PC}$ content (y axis) generated in CLA-TAG and LA-TAG revealed a correlation coefficient of $\geq 0.9$ for both cases. Thus, the increase in PC content was closely related to the increase in polymer content. More specifically, the higher the temperature, the greater was the production of polymers, and this is considered to be the main cause of oxidative degradation.

Furthermore, while the whole polymer contents in CLATAG and LA-TAG after heating to $180^{\circ} \mathrm{C}$ for $3 \mathrm{~h}$ were similar at $55.7 \%$ and $55.4 \%$, respectively, the proportion of polymers with a higher degree of polymerization produced during GPC retention time (Rt): 8.0-10.5 min was $45.7 \%$ for CLA-TAG and $40.5 \%$ for LA-TAG. Therefore, it was considered that high-molecular-weight polymers are produced at a higher frequency in the presence of CLA-TAG through not only the radical oxidation polymerization but also Diels-Alder polymerization.

3.4.2 Thermal oxidation test II 
Examination of the changes over time in polymer content and PC content in each of CLN-TAG or LN-TAG heated to $60-180^{\circ} \mathrm{C}$ showed that the level of polymers production was higher in CLN-TAG than in LN-TAG.

An examination of the correlation between the polymer content and PC content produced in CLN-TAG and LNTAG conducted as described in section 3.4.1 revealed correlation coefficients of 0.8-0.9 at temperatures other than $60^{\circ} \mathrm{C}$. The finding that the gradient a of each straight line, as an indicator of correlation was 1 or higher showed that PC contained substances other than polymers, whereas the finding that the higher the temperature, the smaller the gradient revealed that the percentage of polymer content increased as temperature rose. Furthermore, the polymer contents in CLN-TAG and LN-TAG after heating to $180^{\circ} \mathrm{C}$ for $3 \mathrm{~h}$ were $63.9 \%$ and $49.1 \%$, respectively. A comparison of the degree of polymerization of each peak component showed that in CLN-TAG, the content of polymers with greater degree of polymerization produced during GPC Rt: 8.0-10.5 min accounted for $52.9 \%$ of the total polymers, whereas in LN-TAG the content of such polymers accounted for $33.9 \%$ of the total polymers. Thus, more high-molecular-weight polymers are produced in CLN-TAG. These findings are observed undoubtedly in higher conjugated oil than in lower conjugated oil because oils with higher conjugation degree could react with oxygen molecule or each other easily to form higher-molecule-weight polymer.

\subsection{Establishment of antioxidant techniques for conjugated oils}

In antioxidant tests I and II, PE with $96.9 \%$ purity isolated from soybean phospholipids, and commercially available $\mathrm{AP}$ and $\mathrm{CA}$ were used as synergistic additives to Toc.

\subsubsection{Antioxidant test I}

Comparison of the changes with time of PV, AnV, PC content, and Toc residual ratio in CLA-TAG in non-aqueous autoxidation test, between samples with and without the addition of Toc, revealed that with the addition of 500-1,500 ppm Toc the increase in PV was attenuated, which verifies the antioxidant effect of Toc on CLA-TAG. Samples without added Toc solidified after $960 \mathrm{~h}$, whereas those that were treated with 500, 1,000, and 1,500 ppm Toc did not show solidification until 4,416 h, 5,256 h, and 5,424 h, respectively. Furthermore, in the samples with addition of Toc, the increases in $\mathrm{AnV}$ and PC content were blocked, which verifies the antioxidant effect of Toc on CLA-TAG.

In addition, examination of the changes with time in polymer content, $\mathrm{PC}$ content and the induction period in the Rancimat (CDM) test at $100^{\circ} \mathrm{C}$ after the adding $0-2,000$ ppm of Toc to CLA-TAG showed suppression of the production of polymers and $\mathrm{PC}$ and extension of the induction period as shown in the upper part of Fig. 1

These results support the antioxidant effect of Toc on CLA-TAG. The addition of 500 ppm Toc increased 1.8-fold the induction period, while the addition of 1,000-2,000 ppm Toc, increased about 2.5 -fold the induction period, as compared to that before the addition of Toc. However, in the latter case, there was no significant difference between the induction periods.

For CLN-TAG without the addition of Toc, sampling became impossible due to the comprehensive polymerization that occurred after heating for $6 \mathrm{~h}$, although the suppression of production of polymers and $\mathrm{PC}$ by the addition of Toc could be corroborated. However, there was no significant difference in oxidative behavior among the samples that were supplemented with Toc in the 1,000-2,000

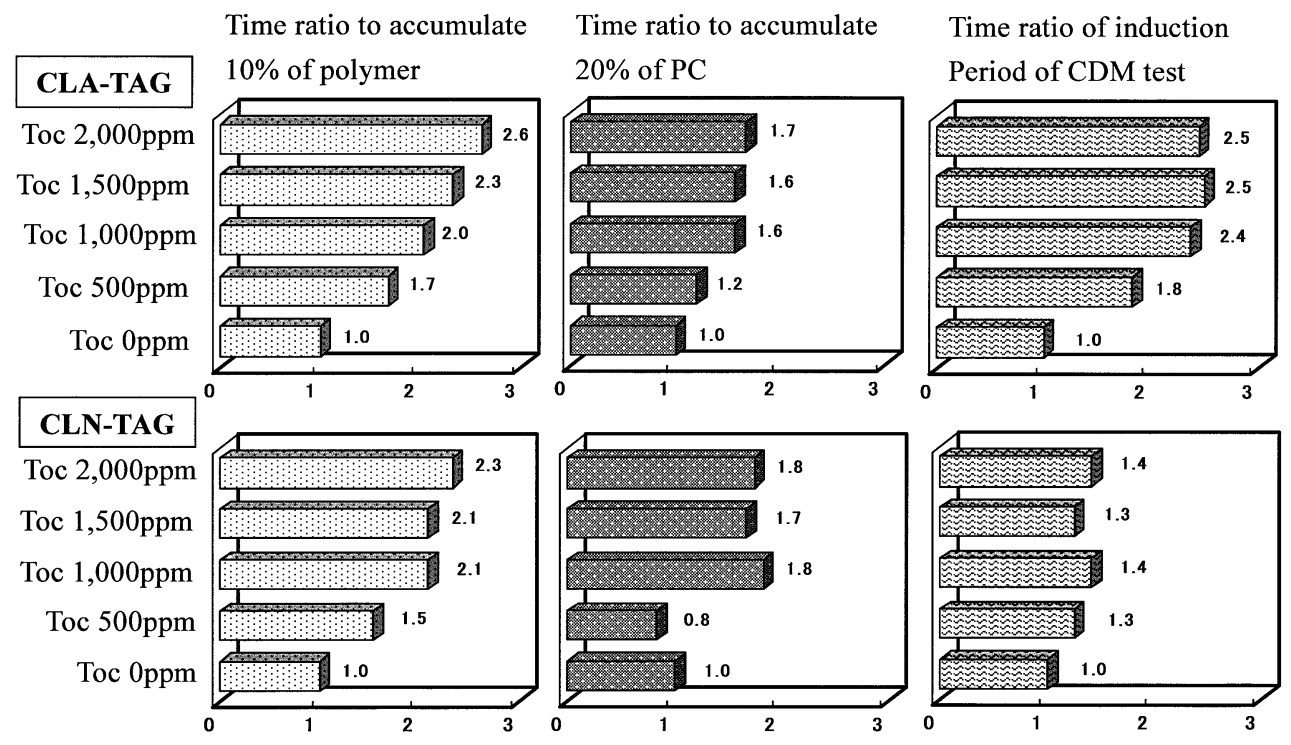

Fig. 1 Antioxidant Effect of Tocopherol on Conjugated Oils in Thermal Oxidation. 


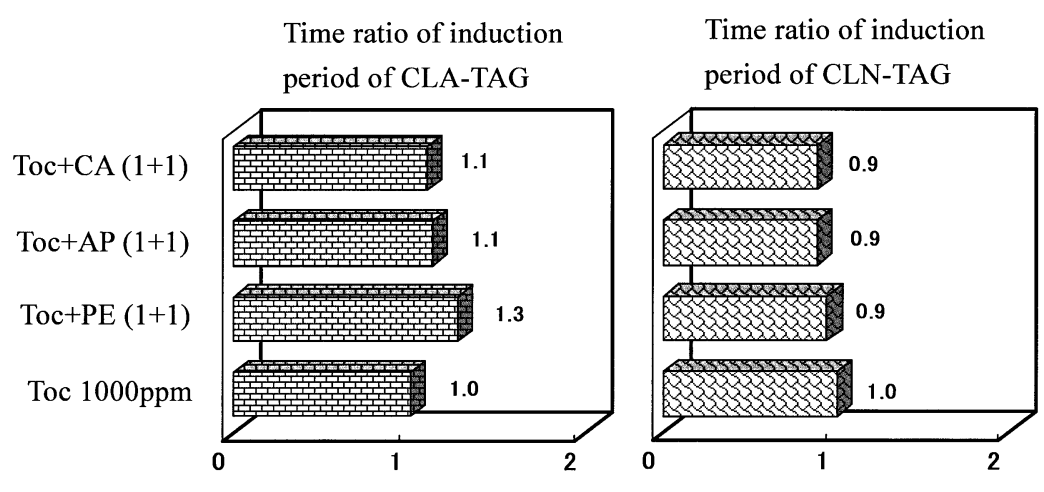

CA: Citric Acid, AP: Ascorbyl Palmitate, PE: Phosphatidyl Ethanolamine

Fig. 2 Synergistic Effects of CA, AP and PE on Tocopherol in Thermal Oxidation of Conjugated Oils, as Evaluated by CDM Test at $100^{\circ} \mathrm{C}$.

ppm range. Accordingly, we considered the addition of 1,000 ppm of Toc to CLN-TAG as optimal, judging from the induction period of CLN-TAG following the addition of $1,000 \mathrm{ppm}$ of Toc as shown in the lower part of Fig. 1

Incidentally, the coefficient of variation for all samples was less than $10 \%$ in triplicate measurement. Therefore, we considered the $1,000 \mathrm{ppm}$ to be the optimal concentration of Toc to be added to both of CLA-TAG and CLN-TAG. 3.5.2 Antioxidant test II

Based on the results presented in section 3.5.1, 1,000 ppm Toc was added to CLA-TAG and CLN-TAG, and each of $\mathrm{PE}, \mathrm{AP}$, and $\mathrm{CA}$ was mixed therein at a molar ratio of Toc to synergistic additive of $1: 1$, to evaluate the antioxidant effect.

Examination of the changes with time in polymer content and PC content and the induction period of CDM test showed that the addition of synergistic additive had no effect on the levels of polymers and PC produced in CLATAG plus 1,000 ppm Toc, whereas in CDM test, the induction period was slightly longer in CLA-TAG with the addition of synergistic additive than in CLA-TAG with 1,000 ppm Toc, and in CLN-TAG, no synergistic effect was observed in any case (Fig. 2), although all coefficient of variations gave less than $10 \%$ in triplicate tests. It is strange why PE did not act as an effective synergist for Toc because PE is very useful synergist for Toc to prevent autoxidation for non-conjugated oils ${ }^{18)}$.

\section{CONCLUSION}

In this study, the behaviors of conjugated oils and nonconjugated oils during autoxidation and thermal oxidation were compared, and the antioxidant effect of Toc on conjugated oils and the effect of synergistic additives on Toc were also examined.

The results of non-aqueous autoxidation test show that conjugated oils are solidified more rapidly than non-conjugated oils upon autoxidation and show higher production of carbonyl compounds and $\mathrm{PC}$ in which polymer was contained, presumably because conjugated oils produce: 1 ) hydroperoxides upon oxidation; 2) another type of peroxide such as a cyclic peroxide and 3) dimer via the Diels-Alder addition reaction. Therefore, we believed that it is necessary to evaluate the autoxidation behaviors of conjugated oils using AnV, PC content and polymer content, since this behavior cannot be assessed accurately using $\mathrm{PV}$ alone.

In both $\mathrm{O} / \mathrm{W}$-type and W/O-type emulsifying autoxidation tests, the increases in $\mathrm{AnV}$ and $\mathrm{PC}$ contents were more pronounced for the conjugated oils than for the nonconjugated oils, in similarity to the results of the non-aqueous autoxidation test.

In thermal oxidation test, $\mathrm{PC}$ and polymer content increased to a greater extent in the conjugated oils than in non-conjugated oils, which confirms that the polymerization reaction has priority over decomposition reaction. Furthermore, in non-conjugated oils, polymers were produced at $150^{\circ} \mathrm{C}$ or above, whereas in conjugated oils, polymers were produced at $90^{\circ} \mathrm{C}$ or above. These results indicate that conjugated oils have a high degree of polymerization.

In the present study, the antioxidant effect of Toc on conjugated oils is confirmed by the autoxidation tests, and the optimal level of Toc supplementation is defined as 1,000 $\mathrm{ppm}$. In addition, the results of $\mathrm{CDM}$ test at $100^{\circ} \mathrm{C}$ corroborate the antioxidant effect of Toc. However, the synergistic effects of $\mathrm{PE}, \mathrm{AP}$ or $\mathrm{CA}$ on Toc were negligible in the conjugated oils.

Therefore, further study is necessary to investigate effective synergists because addition of high concentration of Toc into conjugated oils is not effectively to prevent oxidative degradation of the oils since it is well known that high concentration of Toc acts as a pro-oxidant. 


\section{References}

1. Ha, Y.L.; Grimm, N.K.; Pariza, M.W. Anticarcinogenes from fried ground beef: Heart-altered derivatives of linoleic acid. Carcinogenesis 8, 1881-1887 (1987).

2. Park, Y.; Storkson, J.M.; Albright, K.J.; Liu, W.; Pariza, M.W. Evidence that the trans-10, cis-12 isomer of conjugated linoleic acid induces body composition changes in mice. Lipids 34, 235-241 (1999).

3. Iwata, T. Functionality of conjugated linoleic acids. Shokuhin Kogyo 4.30. 25 (2003).

4. Koga, T.: Sugano, M.; Takahashi, Y.; Tsutsumi, K.; Ishii, T.; Ohta, H. Estimation of Japanese CLA intake from dietary foods. The $41^{\text {st }}$ Annual Meeting of Jpn. Oil Chem. Soc., Abstracts. p.226 (2002).

5. Yanagita, T.; Nagao, K. Anti-obesity and anti-hyperlipidemia of conjugated linoleic acid and its mechanism of action. J. Jpn. Soc. Study Obesity. 9, 194-196 (2003).

6. Nagao, K.; Yanagita, T. Physiological functions of conjugated linoleic acid. J. Jpn. Soc. Nutr. Food Sci. 57, 105-109 (2004).

7. Larsen, T.M.; Toubro, S.; Astrup, A. Efficacy and safety of dietary supplements containing CLA for the treatment of obesity: evidence from animal and human studies. J. Lipid Res. 44, 2234-2241 (2003).

8. Miyashita, K. Anticancer effect of conjugated linolenic acids (CLN). Oleoscience 2, 333-338 (2002).

9. Miyazawa, T.; Tsuzuki, T.; Igarashi, M. Anticancer activity of conjugated fatty acids. Shokuhin Kogyo 3.30, 27-29 (2003).

10. Yamada, K. Shokuhin Seibun no Hataraki. Asakura Shoten. pp.96-99 (2004).

11. Nagao, K.; Yanagita, T. Conjugated fatty acids in food and their health benefits. J. Biosci. Bioeng. 100, 152157 (2005).

12. Tanaka, T.; Suzuki, R. Cancer chemoprevention by food components: Colon cancer chemoprevention by seed oils rich in conjugated linolenic acid. J. Jpn. Soc. Complementary Alternative Med.2, 91-100 (2005).

13. Suzuki, R.; Abe, M.; Miyashita, K. Comparative study of the autoxidation of TAG containing conjugated and nonconjugated $\mathrm{C}_{18}$ PUFA. J. Am. Oil Chem. Soc. 81, 563-595 (2004).

14. Lee, J.; Lee, S.; Kim, I.; Jeong, J.; Rhee, C.; Lee, K. Oxidative instability of CLA concentrate and its avoidance with antioxidants. J. Am. Oil Chem. Soc. 80, 807810 (2003).

15. Jham, G.N.; Teels, F.F.F.; Campos, L.G. Use of aqueous $\mathrm{HCl} / \mathrm{MeOH}$ as esterification reagent for analysis of fatty acids derived from soybean lipids. J. Am. Oil Chem. Soc. 59, 132-133 (1982).

16. Standard Method for the Analysis of Fats, Oils and Related Materials (Japan Oil Chem. Soc., ed), Tentative Method (2003), 4-2000, Peroxide Value (Potentiometric Method), 2.3.1-1996, Acid Value, 2.5.3-1996, Anisidine Value,2.5.6-1996, Oxidized Fatty Acids, 16-2005, GPC Method for Lipids Polymer, 2.2.10.1-1996, Kinetic Viscosity, 2.2.1.1-1996, Color (Lovibond Method).

17. Hara, S.; Ogawa, E.; Totani, Y. Evaluation of heat-deteriorated oils (I) -TLC-FID method for determining polar compounds content. J. Oleo Sci. 55, 167-172 (2006).

18. Segawa, T.; Hara, S.; Totani, Y. Antioxidative behavior of phospholipids for polyunsaturated fatty acid of fish oil. J. Jpn. Oil Chem. Soc. 43, 515-519 (1994). 